\title{
Papers
}

\section{Exposure to non-steroidal anti-inflammatory drugs during pregnancy and risk of miscarriage: population based cohort study}

\author{
De-Kun Li, Liyan Liu, Roxana Odouli
}

\begin{abstract}
Objective To evaluate whether prenatal use of non-steroidal anti-inflammatory drugs (NSAIDs) is associated with increased risk of miscarriage. Design Population based cohort study. Prenatal use of NSAIDs, aspirin, and paracetamol (acetaminophen) ascertained by in-person interview.

Setting Kaiser Permanente Medical Care Program, a healthcare delivery system, in the San Francisco area of the United States.

Participants 1055 pregnant women recruited and interviewed immediately after their positive pregnancy test. Median gestational age at entry to the study was 40 days.
\end{abstract}

Main outcome measures Pregnancy outcomes up to 20 weeks of gestation.

Results 53 women $(5 \%)$ reported prenatal NSAID use around conception or during pregnancy. After adjustment for potential confounders, prenatal NSAID use was associated with an $80 \%$ increased risk of miscarriage (adjusted hazard ratio 1.8 (95\% confidence interval 1.0 to 3.2)). The association was stronger if the initial NSAID use was around the time of conception or if NSAID use lasted more than a week. Prenatal aspirin use was similarly associated with an increased risk of miscarriage. However, prenatal use of paracetamol, pharmacologically different from NSAIDs and aspirin, was not associated with increased risk of miscarriage regardless of timing and duration of use.

Conclusion Prenatal use of NSAIDs and aspirin increased the risk of miscarriage. These findings need confirmation in studies designed specifically to examine the apparent association.

\section{Introduction}

Non-steroidal anti-inflammatory drugs (NSAIDs) are among the most widely used drugs in the developed world $^{1-3}$ and are often used by pregnant women. ${ }^{4-6}$ Because of their widespread use, any unintended effect could have serious adverse public health consequences. Gastrointestinal and renal effects are among the most widely studied and recognised adverse effects of NSAIDs, but reproductive risk, especially from
NSAID use in early pregnancy, has not been systematically evaluated. ${ }^{78}$

A recent Danish case-control study linked pharmacy data with birth registry data. ${ }^{9}$ The authors selected women with registry reports of miscarriages as cases and live births as controls. They reported an association between use of prescribed NSAIDs and miscarriage, with odds ratios that ranged from 1.3 (95\% confidence interval 0.9 to 1.9 ) for NSAID use 10-12 weeks before miscarriage to 7.0 (2.8 to 17.7) for NSAID use one week before miscarriage. However, the study had several limitations from the choice of data sources, including incomplete ascertainment of both miscarriages and NSAID use during pregnancy, lack of information on the reasons for NSAID use, and lack of information on important confounders.

We sought to examine the effect of NSAIDs on the risk of miscarriage by analysing existing data from our recently completed, population based, cohort study of risk factors for miscarriage.

\section{Participants and methods}

We conducted a prospective cohort study of the risk factors for miscarriage among members of the Kaiser Permanente Medical Care Program-an integrated healthcare delivery system, including hospitals and outpatient clinics, that contracts exclusively with a single group of physicians to provide all healthcare services to all members of the system-in northern California. Members of the care programme are representative of the underlying population in the service areas. The primary interest of the study was prenatal exposure to magnetic fields, which is described in detail elsewhere. ${ }^{10}$ We performed data analyses for the current study after we learnt of the reported association between NSAID use during pregnancy and miscarriage. ${ }^{9}$ Because paracetamol (acetaminophen) shares many of the indications for use with NSAIDs but has a different underlying pharmacological effect, we conducted a similar analysis for paracetamol use to control potential confounding by indication for use.

\section{Participants}

From 1996 through 1998, any women members of the Kaiser Permanente care programme were eligible for
Division of Research, Kaiser Foundation Research Institute, Kaiser Permanente, 2000 Broadway, Oakland, California 94612, USA De-Kun Li epidemiologist Liyan Liu programmer analyst Roxana Odouli research associate Correspondence to: D-K Li dkl@dor.kaiser.org bmj.com 2003;327:368

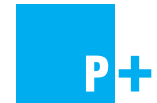

An extra table on bmj.com lists all the indications for prenatal use of the drugs studied. 
the study if they met the following criteria: resided in the San Francisco or south San Francisco area, had a positive pregnancy test, spoke English, and intended to carry the pregnancy to term at the time of recruitment. A document explaining the purpose of the study and the possibility of being contacted for participation in the study was given to every woman who submitted a urine sample for a pregnancy test at San Francisco or south San Francisco facilities. The care programme requires all members who suspect that they are pregnant to have a pregnancy test conducted by the programme's laboratories regardless of whether a home pregnancy test has already been done. A postage-paid, self addressed refusal postcard was included with the document so that women who did not wish to participate in the study could return the card. Our trained female interviewers contacted those who did not return the refusal card to answer their questions about the study and to arrange an interview if they agreed to participate. Among 2729 eligible women, 1063 participated and completed the interview.

\section{Data collection}

We obtained information on use of NSAIDs, aspirin, and paracetamol during pregnancy in an interview conducted soon after each woman's pregnancy was confirmed. We asked the women about their drug use since their last menstruation (since they became pregnant), ascertaining the names of any drugs, the conditions that they were to treat, the timing of their first use, and the duration and frequency of their use. We defined NSAID users as those who reported using ibuprofen or naproxen or preparations containing these drugs (Advil, Motrin, Naprosyn) after their last menstruation. We defined aspirin users as those women who reported using aspirin or preparations containing aspirin (including baby aspirin, Alka Seltzer, Excedrin, and Soma) after their last menstruation. For paracetamol users, we included use of paracetamol or preparations containing it (Tylenol, Tylenol Cold, Tylenol Sinus, Tylenol with codeine, Tylenol PM, Theraflu, Triaminic, Vicodin). We based these definitions on PDR (Physician's Desk Reference) and the drug classification databases published by the American Hospital Formulary Service. In the interviews we also asked the women about their reproductive history, known or potential risk factors for miscarriage, and sociodemographic characteristics.

We ascertained pregnancy outcomes for all participants by searching the Kaiser Permanente care programme inpatient and outpatient databases, reviewing medical records, and contacting those participants whose outcomes could not be determined through the previous two methods. Because miscarriage is defined as natural abortion before 20 weeks of gestation, we obtained information on pregnancy outcomes for every participant up to 20 weeks of gestation.

\section{Statistical analysis}

We used the Cox proportional hazard regression to take into account possible differing gestational ages at entry between the women who used NSAIDs or aspirin and those who did not. ${ }^{11-13}$ By using the Cox model with left truncation, we examined the association between NSAID use and risk of miscarriage at any specific gestational age only among those women who had entered the study and remained pregnant at that specific gestational age. Using the Cox model also allowed us to assess whether the effect of NSAID use on the risk of miscarriage changed with gestational age. The entry time in our study was the gestational age at the positive pregnancy test, and the median gestational age at study entry was 40 days. Data on women who changed their minds and decided to terminate their pregnancy were censored on the date

Table 1 Characteristics of 1055 pregnant women by prenatal use of NSAIDs or aspirin. Values are numbers (percentages)

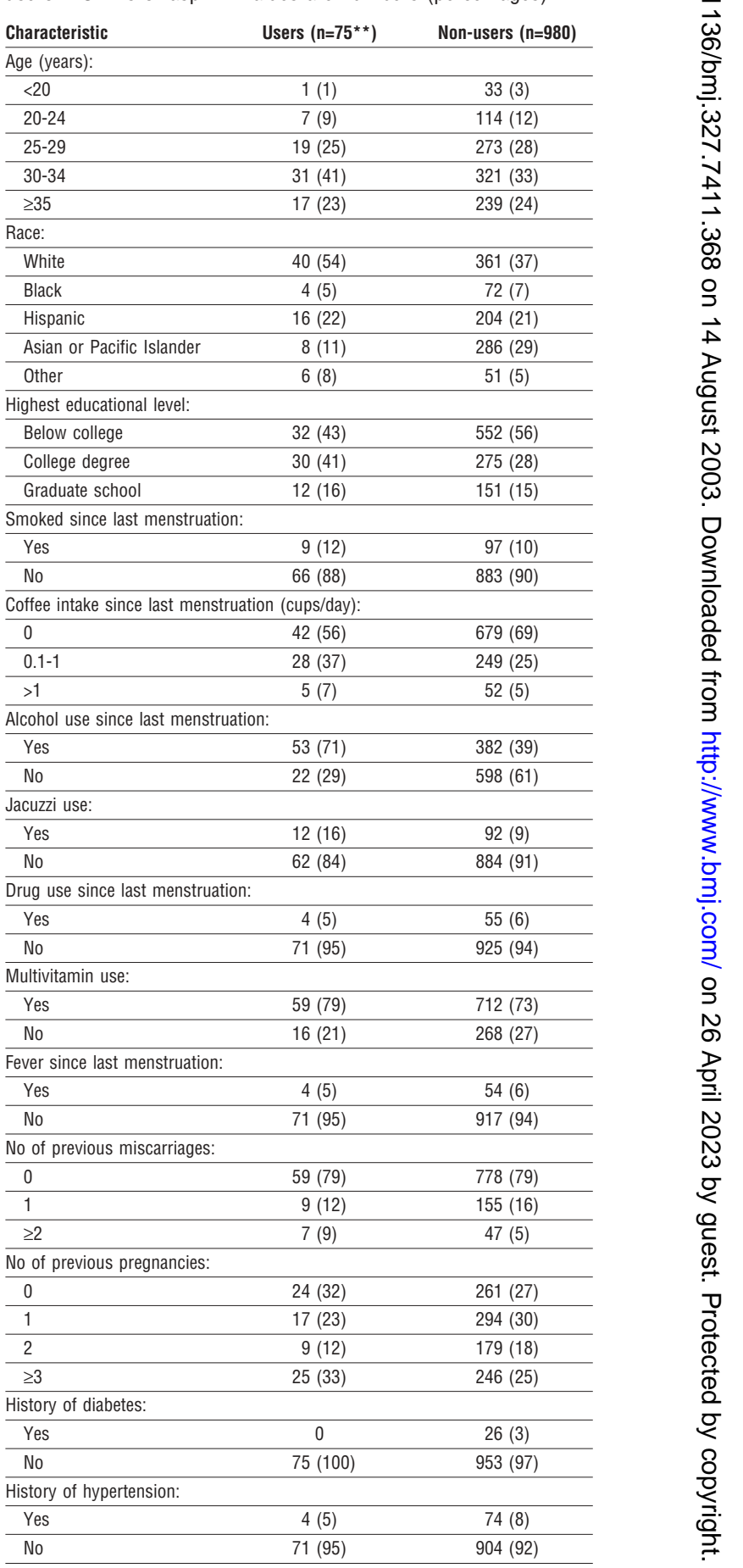

*The number in individual categories may not match the total number owing to missing data.

**53 NSAID users and 22 aspirin users. 
of termination. The variables included in the model for adjustment were potential confounders and known risk factors for miscarriage as well as common socioeconomic and demographic variables.

To avoid the possibility that the condition for which NSAID or aspirin was taken might cause miscarriage, rather than the drugs themselves, we excluded seven women who reported cramping as the reason for taking NSAID or aspirin, because cramping could be an early sign of miscarriage. (Table A on bmj.com lists all the indications for prenatal use of NSAID, aspirin, and paracetamol.) After excluding one further woman with missing data on drug use, we included 1055 women in the final analysis.

\section{Results}

Table 1 shows the background characteristics of NSAID or aspirin users and non-users in our study population. In general, NSAID users had a slightly higher socioeconomic status than non-users and were more likely to be older, white, to have had a college or higher education, and to have had two or more prior miscarriages and more prior pregnancies. Since their last menstruation, they were more likely to have drunk coffee and alcohol, smoked, used a hot tub or Jacuzzi, and taken multivitamins. There was little difference between users and non-users in the frequency of illicit drug use and the occurrence of fever $\geqslant 37.8^{\circ} \mathrm{C}$. None of the NSAID or aspirin users reported having diabetes, and they were less likely than non-users to report having hypertension.

\section{NSAIDs and risk of miscarriage}

After adjustment for previous miscarriage, education, maternal age, gravidity, race, use of a Jacuzzi or hot tub, multivitamin use, and smoking since last menstruation, we found that the use of NSAIDs during pregnancy increased the risk of miscarriage by $80 \%$ (adjusted hazard ratio 1.8 (95\% confidence interval 1.0 to 3.2)). To determine whether NSAID use in early pregnancy presented a particularly high risk of miscarriage, we examined the timing of NSAID use during pregnancy. The risk of miscarriage was much higher when NSAIDs were taken around conception (adjusted hazard ratio 5.6 (2.3 to 13.7)) or were used for longer than a week (8.1 (2.8 to 23.4)) (table 2). Further adjustment for other variables listed in table 1 , including drinking alcohol or coffee in the prenatal period, did not change the results. We obtained similar findings when we used logistic regression (results available on request).

The additional absolute risk of miscarriage associated with NSAID use during pregnancy ranged from $10 \%(25 \%$ minus $15 \%)$ for any NSAID use to $35 \%$ $(50 \%-15 \%)$ for use around conception and to $52 \%$ $(67 \%-15 \%)$ for use for longer than one week (table 2).

\section{Aspirin and risk of miscarriage}

The association of prenatal aspirin use with risk of miscarriage was similar to that observed for prenatal NSAID use, although the association was generally weaker and the estimates unstable because of the small number of aspirin users (table 3 ).

\section{Paracetamol and risk of miscarriage}

Use of paracetamol during pregnancy was not associated with risk of miscarriage regardless of the
Table 2 Prenatal use of NSAIDs by pregnant women and risk of miscarriage. Values are numbers (percentages) unless stated otherwise

\begin{tabular}{|c|c|c|c|}
\hline \multirow[b]{2}{*}{ NSAID use } & \multicolumn{2}{|c|}{ Miscarriage } & \multirow[b]{2}{*}{ Hazard ratio $(95 \% \mathrm{Cl})$} \\
\hline & Yes $(n=162)$ & No $(n=871)$ & \\
\hline Non-users $(n=980) \dagger$ & $149(15)$ & $831(85)$ & 1.0 \\
\hline Users $(n=53)$ : & $13(25)$ & $40(75)$ & 1.8 (1.0 to 3.2$)$ \\
\hline \multicolumn{4}{|l|}{ Gestational age at first use: } \\
\hline At conception $(n=12) \ddagger$ & $6(50)$ & $6(50)$ & 5.6 (2.3 to 13.7$)$ \\
\hline After conception $(n=40)$ & $7(18)$ & $33(83)$ & $1.2(0.5$ to 2.6$)$ \\
\hline \multicolumn{4}{|l|}{ Duration of use: } \\
\hline$\leq 1$ week $(n=47)$ & $9(19)$ & $38(81)$ & $1.3(0.7$ to 2.6$)$ \\
\hline$>1$ week $(n=6)$ & $4(67)$ & $2(33)$ & 8.1 (2.8 to 23.4 ) \\
\hline
\end{tabular}

*Adjusted for previous miscarriage, education, maternal age, gravidity, race, use of Jacuzzi or hot tub, multivitamin use, and smoked since last menstruation. Further adjustment for other variables listed in table 1 did not change the results.

tUsed neither NSAIDs nor aspirin.

fAt conception: within the first week of gestational age.

timing or duration of use (table 4). In this analysis we excluded all NSAID or aspirin users to avoid confounding and, as in the above analyses, also excluded six women who reported cramping as the indication for paracetamol use. In addition, we excluded 41 women who reported using drugs whose paracetamol content could not be unequivocally determined. However, including these 41 women in the calculations did not change the results.

\section{Discussion}

Our analysis of data from a population based prospective cohort study revealed a significantly increased risk of miscarriage associated with prenatal use of NSAIDs

Table 3 Prenatal use of aspirin by pregnant women and risk of miscarriage. Values are numbers (percentages) unless stated otherwise

\begin{tabular}{|c|c|c|c|}
\hline \multirow[b]{2}{*}{ Aspirin use } & \multicolumn{2}{|c|}{ Miscarriage } & \multirow[b]{2}{*}{ Hazard ratio $(95 \% \mathrm{Cl})^{*}$} \\
\hline & Yes $(n=154)$ & No $(n=848)$ & \\
\hline Non-users $(\mathrm{n}=980) \dagger$ & $149(15)$ & $831(85)$ & 1.0 \\
\hline Users $(n=22)$ : & $5(23)$ & $17(77)$ & $1.6(0.6$ to 4.1$)$ \\
\hline \multicolumn{4}{|l|}{ Gestational age at first use: } \\
\hline At conception $(\mathrm{n}=6) \ddagger$ & $3(50)$ & $3(50)$ & 4.3 (1.3 to 14.2$)$ \\
\hline After conception $(n=14)$ & $2(14)$ & $12(86)$ & $1.1(0.3$ to 4.5$)$ \\
\hline \multicolumn{4}{|l|}{ Duration of use: } \\
\hline$\leq 1$ week $(n=16)$ & $3(19)$ & $13(81)$ & $1.4(0.4$ to 4.5$)$ \\
\hline$>1$ week $(\mathrm{n}=5)$ & $2(40)$ & $3(60)$ & $3.0(0.7$ to 12.9$)$ \\
\hline
\end{tabular}

${ }^{*}$ Adjusted for previous miscarriage, education, maternal age, gravidity, race, use of Jacuzzi or hot tub, multivitamin use, and smoked since last menstruation. Further adjustment for other variables listed in table 1 did not change the results.

†Used neither NSAIDs nor aspirin.

$\ddagger$ Within the first week of gestational age.

Table 4 Prenatal use of paracetamol by pregnant women and risk of miscarriage. Values are numbers (percentages) unless stated otherwise

\begin{tabular}{|c|c|c|c|}
\hline \multirow[b]{2}{*}{ Paracetamol use * } & \multicolumn{2}{|c|}{ Miscarriage } & \multirow[b]{2}{*}{ Hazard ratio $(95 \% \mathrm{Cl}) \dagger$} \\
\hline & Yes $(n=141)$ & No $(n=793)$ & \\
\hline Non-users $(\mathrm{n}=762)$ & $117(15)$ & $645(85)$ & 1.0 \\
\hline Users $(n=172)$ : & $24(14)$ & $148(86)$ & 1.2 (0.8 to 1.8$)$ \\
\hline \multicolumn{4}{|l|}{ Gestational age at first use: } \\
\hline At conception $(n=19) \ddagger$ & $2(11)$ & $17(89)$ & $0.8(0.2$ to 3.3$)$ \\
\hline After conception $(\mathrm{n}=147)$ & $21(14)$ & $126(86)$ & $1.2(0.8$ to 2.0$)$ \\
\hline \multicolumn{4}{|l|}{ Duration of use: } \\
\hline$\leq 1$ week $(n=147)$ & $22(15)$ & $125(85)$ & $1.3(0.8$ to 2.1$)$ \\
\hline$>1$ week $(n=23)$ & $2(9)$ & $21(91)$ & 0.7 (0.2 to 2.9$)$ \\
\hline
\end{tabular}

*NSAID or aspirin users were excluded from this analysis.

†Adjusted for previous miscarriage, education, maternal age, gravidity, race, use of Jacuzzi or hot tub, multivitamin use, and smoked since last menstruation. Further adjustment for other variables listed in table 1 did not change the results.

$\ddagger$ Within the first week of gestational age. 
or aspirin. The associated risk was particularly high when NSAIDs were used around the time of conception, consistent with the hypothesis that prostaglandin inhibition by NSAIDs interferes with implantation (see below). The risk was also higher when NSAIDS were used for longer than a week, which indicates a dose-response relationship.

\section{Strengths and limitations of study}

Our study is a substantial improvement on the study that first reported an association between NSAID use and miscarriage. ${ }^{9}$ Our ascertainment of NSAID and aspirin exposure was more complete and based on actual use rather than prescription, and our ascertainment of miscarriage was complete among all participants. We also identified indications for use of the drugs, controlled for confounding factors, evaluated the effect of timing and duration of use, and had an internal comparison group of paracetamol users.

However, because examination of the association of NSAID use with miscarriage was not the main aim of the original study, this study has potential limitations. Despite direct questioning of all participants, our ascertainment of NSAID and aspirin use during pregnancy was still probably incomplete. Because our study was a prospective cohort study, most of the participants were still pregnant when they were interviewed. Therefore, some of the participants who reported no use could have used the drugs after the interview and while still pregnant. However, this misclassification of some users into the non-user group would, if anything, have attenuated the observed association of NSAID and aspirin use with miscarriage. In addition, the reported frequency of NSAID use in our study $(5 \%)$ was much higher than that reported in the previous study $(1 \%),{ }^{9}$ indicating a more complete ascertainment of NSAID use. Finally, the risk of miscarriage was mainly associated with NSAID use around conception, which we should have completely ascertained for all participants.

\section{Probable mechanism of effect}

The association between NSAID use and miscarriage is unlikely to be due to the underlying indications for use of NSAIDs or aspirin. Paracetamol, which shares many of the indications for use with NSAIDs (see table A on bmj.com) but has a different underlying pharmacological effect, had no effect on risk of miscarriage. NSAIDs and aspirin are considered to inhibit prostaglandin biosynthesis in most organ systems, whereas paracetamol inhibits prostaglandin biosynthesis only in the central nervous system.

Animal studies have shown that prostaglandins are needed for successful implantation of an embryo into the uterus wall. ${ }^{8}$ Prostaglandins have also been reported to play an important role in human ovulation and implantation through their own effect and interaction with platelet activating factors and cytokines, both in the uterus and in the embryo. ${ }^{14-16}$ Suppression of prostaglandin biosynthesis by NSAIDs in peripheral tissues, including those of the reproductive system, could therefore lead to abnormal implantation that predisposes an embryo to miscarriage. In fact, the newer selective NSAIDs (cyclo-oxygenase 2 inhibitors) are classified as pregnancy category $\mathrm{C}$ because of increased peri-implantation and post-implantation losses and reduced fetal survival in rats and rabbits.

\section{What is already known on this topic}

A recent study reported an increased risk of miscarriage with NSAID use during pregnancy, but the study was limited, relying solely on linkage of data from registers

\section{What this study adds}

This population based cohort study provides stronger evidence of an association between NSAID and aspirin use and miscarriage

The risk of miscarriage was highest with NSAID use around the time of conception increases and with NSAID use for longer than a week

Paracetamol, which shares many of the indications for use with NSAIDs but has a different underlying pharmacological effect, had no effect on risk of miscarriage

However, such an effect with non-selective NSAIDs has not been as well examined.

In addition, it has been suggested that a delicate balance of the concentration of various types of prostanoids is essential for maintaining normal blood pressure during pregnancy. ${ }^{8}$ NSAID use that suppresses the production of prostaglandins may have an adverse effect on placental perfusion and circulation. Without a healthy placenta, the risk of fetal demise can increase greatly.

We found that the highest risk of miscarriage was with NSAID use around the time of conception. This supports the mechanism of normal implantation being altered by NSAID use.

\section{Conclusions}

If NSAID use during pregnancy, especially around conception, is associated with miscarriage it will have wide clinical implications because many women are likely to be prescribed NSAIDs during the periconceptional period. Our findings will need confirmation in studies that are designed specifically to examine the association between NSAID use and the risk of miscarriage. Meanwhile, however, it may be prudent for physicians and women who are planning to be pregnant to be aware of this potential risk and avoid using NSAIDs around conception.

Contributors: D-KL conceived of and designed the study and analysed and interpreted the data. LL helped analyse and interpret the data. RO helped design the study and collected the data. $\mathrm{D}-\mathrm{KL}$ is the guarantor of this paper and took full responsibility for the conduct of the study, had access to the data, and controlled the decision to publish.

Funding: The study was supported in part by funds from the California Public Health Foundation and the National Institute of Child Health and Human Development (NICHD).

Competing interests: None declared.

Ethical approval: The Institutional Review Board of Kaiser Permanente approved the protocol and conduct of the study.

1 Setter SM, Corbett C, Gates BJ, Terriff C, Johns CA, Sclar DA, et al. Nonsteroidal anti-inflammatory drugs: The need for assessment and education. Home Care Provid 2001;6:100-5.

2 Brooks P. Use and benefits of nonsteroidal anti-inflammatory drugs. Am J Med 1998;104:9-13S.

Hernandez-Diaz S, Garcia-Rodriguez LA. Epidemiologic assessment of Hernandez-Diaz S, Garcia-Rodriguez LA. Epidemiologic assessment of
the safety of conventional nonsteroidal anti-inflammatory drugs. Am J Med 2001;110(suppl 3A):20S-7S. 
4 Hertz-Picciotto I, Hopenhayn-Rich C, Golub M, Hooper K. The risks and benefits of taking aspirin during pregnancy. Epidemiol Rev 1990;12:

5 Werler MM, Mitchell AA, Shapiro S. The relation of aspirin use during the first trimester of pregnancy to congenital cardiac defects. N EnglJ Med $1989 ; 321: 1639-42$

6 Streissguth AP, Treder RP, Barr HM, Shepard TH, Bleyer WA, Sampson PD, et al. Aspirin and acetaminophen use by pregnant women and subsequent child IQ and attention decrements. Teratology 1987;35:211-9.

7 Janssen NM, Genta MS. The effects of immunosuppressive and anti-inflammatory medications on fertility, pregnancy, and lactation. Arch Intern Med 2000;160:610-9.

8 Dawood MY. Nonsteroidal antiinflammatory drugs and reproduction. $A m$ J Obstet Gynecol 1993;169:1255-65.

9 Nielsen GL, Sorensen HT, Larsen H, Pedersen L. Risk of adverse birth outcome and miscarriage in pregnant users of non-steroidal antiinflammatory drugs: population based observational study an case-control study. BMJ 2001;322:266-70.

10 Li DK, Odouli R, Wi S, Janevic T, Golditch I, Bracken TD, et al. A population-based prospective cohort study of personal exposure to mag- netic fields during pregnancy and the risk of miscarriage. Epidemiology 2002;13:9-20.

11 Anderson PK, Gill RD. Cox's regression model for counting processes: a large sample study. Ann Stat 1982;10:1100-20.

12 Therneau TM, Grambsch PM. Modeling survival data: extending the Cox model. New York: Springer-Verlag, 2000.

13 Howards PP, Hertz-Picciotto I. Spontaneous abortion and left truncation [abstract] Am J Epidemiol 2002;155(11):S35.

14 Van der Weiden RM, Helmerhorst FM, Keirse MJ. Prostanoid excretion in incipient singleton and twin pregnancies. Am J Obstet Gynecol 1996;174:1614-7.

15 Van der Weiden RM, Helmerhorst FM, Keirse MJ. Influence of prostaglandins and platelet activating factor on implantation. Hum Reprod 1991;6:436-42.

16 Van der Weiden RM, Wouters JM. Infertility may sometimes be associated with non-steroidal anti-inflammatory drug consumption. BrJ Rheumatol 1997;36:605

(Accepted 2 June 2003) 